\title{
Characterization of biofilm formation, antimicrobial resistance, and staphylococcal cassette chromosome mec analysis of methicillin resistant Staphylococcus hominis from blood cultures of children
}

\author{
Setareh Soroush ${ }^{[1]}$, Fereshteh Jabalameli ${ }^{[1]}$, Morovat Taherikalani ${ }^{[2]}$, \\ Mohammad Amin Eslampour ${ }^{[3]}$, Reza Beigverdi ${ }^{[1]}$ \\ and Mohammad Emaneini[ ${ }^{[1]}$
}

[1]. Department of Microbiology, School of Medicine, Tehran University of Medical Sciences, Tehran, Iran. [2]. Department of Microbiology, School of Medicine, Lorestan University of Medical Sciences, Khoramabad, Iran. [3]. Department of Theriogenology, Science and Research Branch, Islamic Azad University, Tehran, Iran.

\begin{abstract}
Introduction: Methicillin resistant Staphylococcus hominis (MRSHo) has been recognized as an important human pathogen, particularly in immunocompromised patients. Methods: A total of 19 S. hominis isolates were collected from children at the Children's Medical Centre, Tehran, Iran, from March 2012 to February 2013. MRSHo susceptibility against 13 antimicrobial and 3 antiseptic agents was determined using disk diffusion (DAD) and minimum inhibitory concentration (MIC), respectively. All isolates were subjected to polymerase chain reaction (PCR) assay for 15 distinct resistance genes, staphylococcal cassette chromosome mec (SCCmec), and arginine catabolic mobile elements (ACMEs). Biofilm production of the isolates was determined using a colorimetric microtiter plate assay. Results: Of the 19 isolates, 16 were resistant to oxacillin and harbored mecA. High resistance was also observed against trimethoprim/sulfamethoxazole (81.2\%). All MRSHo isolates were susceptible to the three disinfectants tested (Septicidine-PC, Septi turbo, and Sayacept-HP). In total, 15 (78.9\%) isolates produced biofilms. Three isolates had SCCmec types (V and VIII), 13 were untypable (UT), and 5 had ACME type II. Conclusions: The results indicate that MRSHo with high antibiotic resistance and unknown SCCmec might become a serious problem in the future for the treatment of patients such as children.
\end{abstract}

Keywords: MRSHo. SCCmec. ACME. Biofilm.

\section{INTRODUCTION}

Staphylococcus hominis, a member of coagulase-negative staphylococci (CoNS) found asymptomatically on the skin, arms, legs, and surfaces of the axilla, is an opportunistic pathogen capable of causing a wide variety of diseases including bacteremia, septicemia, and endocarditis especially in immunocompromised patients ${ }^{1,2}$. A growing concern is the emergence of methicillin resistant Staphylococcus hominis (MRSHo), which is the third most common pathogen among methicillin resistant CoNS (MRCoNS) in clinical isolates ${ }^{3,4}$. Resistance to methicillin is due to the acquisition of mecA, which encodes PBP2a - a transpeptidase with a low affinity for beta-lactam antibiotics such as penicillin. mecA is located

Corresponding author: Dr. Mohammad Emaneini.

e-mail: emaneini@tums.ac.ir

Received 19 September 2016

Accepted 27 April 2017 on the staphylococcal cassette chromosome mec (SCCmec). Eleven main types of SCCmec differing in size and genetic composition have been assigned for staphylococcal isolates ${ }^{5}$. The capability of CoNS, including S. hominis, to produce disease depends on their ability to create a wide variety of virulence factors that contribute to colonization and disease incidence in hosts, such as biofilm production and the arginine catabolic mobile element (ACME) ${ }^{6}$. Arginine catabolic mobile elements includes two gene clusters, arc encoding a secondary arginine deiminase system and opp-3 encoding an ABC transporter ${ }^{6}$. In recent years, the widespread use of chemical disinfectants (Septicidine-PC, Sayacept-HP, and Septi turbo) in hospital settings played a critical role in the emergence of disinfectant-resistant microorganisms. Despite the incidence of methicillin resistance among staphylococci, there is currently little information available on the incidence and types of these resistances in many countries, such as Iran. The aim of this study was to investigate the antibiotic and antiseptic resistance patterns and prevalence of different SCCmec and ACME types among $S$. hominis isolates obtained from Iranian children. 


\section{METHODS}

\section{Bacterial isolates}

From March 2012 to February 2013, 256 CoNS strains were isolated from children at the Children's Medical Center, Tehran. Only one strain for each patient was selected. Isolates were identified as S. hominis, using conventional methods confirmed by the targeting of $n u c^{7,8}$. In these isolates, the gene encoding methicillin resistance $(m e c A)$ was targeted by polymerase chain reaction $(\mathrm{PCR})^{9}$.

\section{Susceptibility testing and amplification of resistance genes}

Antimicrobial susceptibility was determined using the disk diffusion method on Mueller-Hinton agar based on the Clinical and Laboratory Standards Institute (CLSI) guidelines ${ }^{10}$. The following antibiotics (Mast Co., UK) were tested: ciprofloxacin $(5 \mu \mathrm{g})$, clindamycin $(2 \mu \mathrm{g})$, erythromycin $(15 \mu \mathrm{g})$, gatifloxacin $(5 \mu \mathrm{g})$, gentamicin $(10 \mu \mathrm{g})$, linezolid $(30 \mu \mathrm{g})$, mupirocin $(200 \mu \mathrm{g})$, quinupristin/dalfopristin $(15 \mu \mathrm{g})$, rifampin $(5 \mu \mathrm{g})$, tetracycline $(30 \mu \mathrm{g})$, tigecycline $(15 \mu \mathrm{g})$, and trimethoprim/sulfamethoxazole $(1.25 / 23.75 \mu \mathrm{g})$. In addition, inducible clindamycin resistance was checked by placing clindamycin and erythromycin disks (D-shaped) on the agar, 15-26mm apart. The minimum inhibitory concentrations (MICs) of oxacillin and vancomycin were determined using the broth micro-dilution method according to CLSI guidelines ${ }^{10}$. Strains that were resistant to three or more antimicrobial classes were considered to possess multiple drug resistance (MDR) ${ }^{11}$.

\section{Minimum inhibitory concentrations of disinfectants}

Commonly used disinfectants in the Children's Medical Centre were obtained in commercial preparations: SepticidinePC contains $0.5 \%(\mathrm{w} / \mathrm{w})$ chlorhexidine digluconate and $75 \%$ (w/w) alcohol; Sayacept-HP contains $0.25 \%(\mathrm{w} / \mathrm{w})$ quaternary ammonium compounds (QACs) and biguanide; and Septi turbo contains 70\% (w/w) isopropanol and $0.25 \%$ (w/w) QACs (all supplied by Behban Shimi Pharmaceutical Co., Gorgan, Iran). Although these disinfectants should be used without dilution, their MICs were determined by serial 2-fold micro-dilution (1- to 16-fold) in Mueller-Hinton broth (Conda, Spain), and the addition of the reducing dye triphenyl tetrazolium chloride (TTC) (Sigma Aldrich, USA) ${ }^{12}$. Each microdilution was inoculated with $50 \mu \mathrm{L}$ of $1.5 \times 10^{6}$ colony-forming units per milliliter $(\mathrm{CFU} / \mathrm{mL})$ of the overnight cultures in a brain heart infusion (BHI) blood agar base. The microplates were then incubated at $37^{\circ} \mathrm{C}$ for $24 \mathrm{~h}$, and the lowest concentration of the disinfectant that completely inhibited the growth of the microorganisms was recorded as the MIC. Staphylococcus aureus ATCC 29213 was used as a control. Antibiotic-resistance genes, namely, blaZ, erm $A$, ermB, erm $C, \operatorname{msr} A, \operatorname{msr} B$, tetM, tetK, $\operatorname{aac}\left(6^{\prime}\right)-I e-\operatorname{aph}\left(2^{\prime \prime}\right)-I a, \operatorname{aph}\left(3^{\prime}\right)-I I I a, \operatorname{ant}\left(4^{\prime}\right)-I a$, mupA, and vanA, and antiseptic-resistance genes, namely, smr and qac $A / B$, were screened by PCR and multiplex-PCR methods ${ }^{13,14}$.

\section{Biofilm production}

The capacity of isolates for biofilm production was determined using a colorimetric microtiter plate assay ${ }^{15}$. Briefly,
MRSHo isolates were grown on Trypticase Soy Broth (TSB) overnight at $37^{\circ} \mathrm{C}$ (Merck, Darmstadt, Germany), and the cultures were then diluted 1:100 in TSB medium. Consequently, $150 \mu \mathrm{L}$ of this cell suspension was used to inoculate sterile flat-bottomed 96-well polystyrene microtiter plates. After 24 $\mathrm{h}$ incubation at $37^{\circ} \mathrm{C}$, without shaking, the wells were gently washed three times with $200 \mu \mathrm{L}$ of phosphate buffered saline (PBS; Sigma-Aldrich, USA) and dried in an inverted position. For fixation of biofilms, $100 \mu \mathrm{L}$ of $99 \%$ methanol was added and after $15 \mathrm{~min}$, supernatants were removed and the plate was air-dried. Next, $100 \mu \mathrm{L}$ of $1 \%$ crystal violet (CV; HiMedia, India) was added to all the wells. Excess $\mathrm{CV}$ was removed after $20 \mathrm{~min}$ by washing the plate under running tap water. Bound CV was released by adding $150 \mu \mathrm{L}$ of $33 \%$ acetic acid. The optical density (OD) of each well was measured at $590 \mathrm{~nm}$ using a microtiter plate reader. All the tests were repeated three times. Uninoculated medium was used as a control to determine the background OD. The cut-off OD (ODc) was defined as three standard deviations above the mean OD of the negative control, and the final OD value of a tested strain was defined as the average OD of the strain reduced by the ODc value. The adherence ability of the tested strain was classified into four categories based on the $\mathrm{OD}^{15}$ : non-adherent (OD $<\mathrm{ODc}$ ), weakly adherent (ODc $<$ OD $<2 X O D c$ ), moderately adherent (2XODc $<$ OD $<4$ XODc), and strongly adherent (4XODc $<$ OD). The genes encoding intracellular adhesion (icaA and icaD) were targeted by PCR using the primers as previously described ${ }^{9}$.

\section{Staphylococcal cassette chromosome mec and arginine catabolic mobile element typing}

The primers used to detect SCCmec types and their variants, the conditions of PCR, and the size of the amplified products were each used as described by Ito et al. ${ }^{16}$ The ACME typing PCR was performed with template deoxyribonucleic acid (DNA) from each isolate to determine if they harbored parts of ACME genes I to $\mathrm{III}^{17}$.

\section{RESULTS}

Of the 256 CoNS strains, 19 (7.4\%) were $S$. hominis; 16 $(84.2 \%)$ of which were resistant to oxacillin $\left(\mathrm{MIC}_{90}=32 \mu \mathrm{g} / \mathrm{mL}\right)$ and contained mecA. These $16 \mathrm{MRSHo}$ isolates were obtained from blood cultures from the following wards: emergency (5), oncology (2), ophthalmology (2), neonatal intensive care unit (2), interior (2), nephrology (1), infection (1), and unknown (1). The phenotypic and genotypic characterizations of MRSHo isolates are shown in Table 1. All isolates were susceptible to linezolid, quinupristin/dalfopristin, and rifampin and had intermediate susceptibility to vancomycin $\left(\mathrm{MIC}_{90}=4 \mu \mathrm{g}\right.$ / $\mathrm{mL})$; no vanA was detected. High resistance rates were found against trimethoprim/sulfamethoxazole $(81.2 \%)$, erythromycin $(75 \%)$, and tetracycline (62.5\%). Isolates were also resistant to gentamicin $(25 \%)$, ciprofloxacin $(25 \%)$, clindamycin $(25 \%)$, mupirocin $(18.7 \%)$, gatifloxacin $(12.5 \%)$, and tigecycline $(12.2 \%)$. All isolates were susceptible to the tested disinfectants, and the MICs of Septicidine-PC, Septi turbo, and SayaceptHP were 8-, 8-, and 14-fold lower than the concentration recommended for use by the manufacturers (Table 1). 


\section{TABLE1}

Antibiotic and disinfectant resistance and biofilm production of MRSHo.

\begin{tabular}{|c|c|c|c|c|c|c|c|c|}
\hline \multirow[b]{2}{*}{ Isolates } & \multirow[b]{2}{*}{ Ward } & \multicolumn{2}{|c|}{$\operatorname{MIC}(\mu \mathrm{g} / \mathrm{mL})$} & \multicolumn{3}{|c|}{$\begin{array}{l}\text { MIC (dilution fold) } \\
\text { of antiseptics }\end{array}$} & \multirow[b]{2}{*}{ Biofilm } & \multirow[b]{2}{*}{ Genes } \\
\hline & & OXA $^{*}$ & VAN & SEP ${ }^{\mathrm{s}}$ & SYA & TOR & & \\
\hline 2 & $\mathrm{NICU}^{*}$ & 32 & 2 & 10 & 16 & 10 & $\mathrm{~W}$ & Ie-aph(2)-Ia, ant(4)-Ia, tetk, blaz, mup, msrA \\
\hline 5 & INT & 8 & 4 & 9 & 15 & 8 & $\mathrm{~W}$ & tetk \\
\hline 6 & OP & 16 & 4 & 11 & 15 & 9 & $\mathrm{~W}$ & tetm, blaz \\
\hline 7 & NICU & 32 & 2 & 9 & 15 & 8 & $\mathrm{~W}$ & Ie-aph(2)-Ia, tetk, blaz \\
\hline 11 & EMR & 64 & 4 & 9 & 16 & 10 & $\mathrm{~W}$ & $a a c(6)-I e-a p h(2)-I a, a p h(3)-I I I a, b l a z, m s r A$ \\
\hline 12 & EMR & 128 & 4 & 8 & 15 & 8 & $\mathrm{~W}$ & Ie-aph(2)-Ia, ant(4)-Ia, tetk, blaz, тир, msrA, qacAB \\
\hline 13 & EMR & 4 & 2 & 9 & 15 & 10 & $\mathrm{~W}$ & tetk, msrA \\
\hline 14 & $\mathrm{ONC}$ & 16 & 2 & 9 & 14 & 8 & $\mathrm{~W}$ & tetk, blaz, msrA \\
\hline 15 & EMR & 16 & 4 & 10 & 15 & 8 & W & blaz \\
\hline 16 & INT & 4 & 8 & 9 & 15 & 9 & $\mathrm{~N}$ & blaz, msrA \\
\hline
\end{tabular}

MRSHo: methicillin resistant Staphylococcus hominis MIC: minimum inhibitory concentration; " OXA: oxacillin; VAN: vancomycin; ${ }^{\mathbf{s}}$ SEP: Septicidine-PC; SYA: Sayacept-HP; TOR: Septi turbo; UN: unknown; ${ }^{*}$ NICU: neonatal intensive care unit; ONC: oncology; NEPH: nephrology; INT: interior; OP: ophthalmology; INF: infection wards; EMR: emergency; ${ }^{\#} \mathbf{W}$ : weak; M: moderate; N: no biofilm.

$\mathrm{MIC}_{90}$ of strains to Septicidine-PC, Septi turbo, and Sayacept-HP were 8-, 8-, and 15-fold diluted, respectively. The prevalence of resistance genes were as follow: blaZ $(81.2 \%)$, tetK $(75 \%)$, $\operatorname{tetM}(6.2 \%), \operatorname{aac}\left(6^{\prime}\right)-\operatorname{Ie}-\operatorname{aph}\left(2^{\prime \prime}\right)-\operatorname{Ia}(43.7 \%), \operatorname{ant}\left(4^{\prime}\right)-\operatorname{Ia}(18.7 \%)$, $\operatorname{aph}\left(3^{\prime}\right)$-IIIa (25\%), msrA (43.7\%), ermA (6.2\%), mupA (18.7\%), Smr (12.2\%), and qacA/B (6.2\%). Approximately 15 (78.9\%) of the isolates produced biofilms. Among these, $12(80 \%)$ were weakly adherent biofilm producers, and $3(20 \%)$ produced moderately adherent biofilms. icaA and icaD were found in only one of moderate biofilm producers.

Of the 16 oxacillin-resistant MRSHo isolates, only 3 were typed by SCCmec typing: 2 were type VIII and 1 was type V (Table 2). Analysis of the mec and $\mathrm{ccr}$ complexes among the 13 untypable (UT) isolates revealed that four had mec complex A $+c c r C$ (UT1); two, mec complex A + ccrAB1 and $c c r C$ (UT2); one, mec complex B $+c c r C$ (UT3); one, mec complex A and c1 + ccrAB1 (UT4); one, mec complex A and no ccr (UT5); one, only $c c r A B 1$ and $c c r C$ (UT6); three, neither $c c r$ nor mec (UT7). Some of the isolates within the same UT group had different junk yard motifs. In ACME typing, only $\operatorname{arcC}$ was positive in $5(31.2 \%)$ isolates, which were considered ACME type II. All isolates were negative for the opp-3 gene.

\section{DISCUSSION}

In this study, the methicillin resistance rate was slightly higher than that reported in other studies conducted in the USA ( $80-82 \%$ ), Mexico ( $81 \%$ ), and Tunis $(75 \%)^{2-4,18}$, and the MDR rate was higher than that reported in the Tunis study $(94 \%)^{2}$. The different MRSHo rates in different countries may reflect differences in the doses and dosing schedules used and in the spread of particular clones. In the current study, the reduced vancomycin susceptibility observed in some clinical isolates could preclude full resistance, which is in contrast to the findings of Palazzo et al. ${ }^{19}$, who observed $83.3 \%$ of isolates with resistance to vancomycin. In this study, we also observed high levels of resistance to gatifloxacin and tigecycline. Unfortunately, fluoroquinolones, especially ciprofloxacin and gatifloxacin, are widely used for the treatment of various bacterial infections in Iran, which may contribute to the emergence of resistant strains ${ }^{20}$. Moreover, several studies that used MIC methods revealed that $100 \%$ of CoNS were susceptible to tigecycline ${ }^{21-23}$. Given that we used disk diffusion to determine the tigecycline susceptibility of our MRSHo isolates, MIC-based methods might indicate that all of our isolates were susceptible. 
TABLE 2

SCCmec and ACME types of MRSHo isolates.

\begin{tabular}{|c|c|c|c|c|c|c|}
\hline Isolates & mec complex & \multicolumn{2}{|c|}{ SCCmec } & type & \multicolumn{2}{|c|}{ ACME } \\
\hline 1 & A & $\mathrm{A} 1 \mathrm{~B} 1 / \mathrm{A} 4 \mathrm{~B} 4$ & - & $\mathrm{VIII}+\mathrm{AB} 1$ & - & - \\
\hline 2 & $\mathrm{~A} / \mathrm{C} 1$ & $\mathrm{AB} 1$ & - & UT4 & - & - \\
\hline 3 & B & $\mathrm{C}$ & Iva & UT3 & - & - \\
\hline 6 & $\mathrm{~A} / \mathrm{C} 2$ & $\mathrm{C}$ & $\mathrm{Ia} / \mathrm{Iia}$ & $\mathrm{V}+\mathrm{A}$ & $\operatorname{arc} A$ & II \\
\hline 7 & A & $\mathrm{A} 1 \mathrm{~B} 1 / \mathrm{C}$ & - & UT2 & - & - \\
\hline 8 & A & $\mathrm{A} 1 \mathrm{~B} 1 / \mathrm{C}$ & $\mathrm{Ia} / \mathrm{IIa}$ & UT2 & $\operatorname{arcA}$ & II \\
\hline 9 & - & $\mathrm{A} 1 \mathrm{~B} 1 / \mathrm{C}$ & Iva/IIa/Ivc & UT6 & - & - \\
\hline 13 & - & - & - & UT7 & - & - \\
\hline 14 & - & - & - & UT7 & $\operatorname{arc} A$ & II \\
\hline 15 & $\mathrm{~A}$ & - & - & UT5 & - & - \\
\hline 16 & A & $\mathrm{C}$ & Ia & UT1 & $\operatorname{arcA}$ & II \\
\hline
\end{tabular}

SCCmec: Staphylococcal cassette chromosome mec; ACME: arginine catabolic mobile element; MRSHo: methicillin resistant Staphylococcus hominis ; untypable (UT) 1: $m e c$ complex A + $c c r C$; UT2: mec complex A + ccrAB1 and $c c r C$; UT3: mec complex B + ccrC; UT4: mec complex A and c1 + $c c r A B 1$; UT5: mec complex A - $c c r$; UT6: only $c c r A B 1$ and $c c r C$; UT7: neither $c c r$ nor mec genes.

In the current study, some strains with or without resistance genes ( $s m r$ and $q a c A / B$ ) had the same MIC, which may be owing to the presence of other resistance genes. In a Norway study that worked on cattle and goats, $s m r$ was detected in one of two $S$. hominis isolates, which is higher than our results in humans ${ }^{24}$. With regards to biofilm production, our results were similar to those obtained in certain studies conducted in Argentina and Mexico $^{25,26}$; however, they contrasted with that of a different Mexico study ${ }^{4}$, which reported high biofilm production in most strains Therefore, the low and moderate biofilm production may be related to factors other than the presence of ica. Given that different ACME types help to maintain $S$. epidermidis in the environment, the presence of ACME type II in some of these strains may improve their ability to remain and colonize. The SCCmec types observed in our study have previously been found in the USA (type VIII), China (type VIII and V), and Tunis (type VIII); nevertheless, none of these studies reported additional segments with these types ${ }^{2,3,27}$. The two strains in our study with SCCmes type VIII (strain 1 and 12) exhibited approximately the same antibiotic resistance (MDR) and biofilm production (weak), but strain 12 had $q a c A / B$ and an extra $c c r C$ gene; these two strains were identified with an interval of 10 months. A combination of mec complex class A with $c c r$ type 5 and mec complex class A with $c c r$ type 1 has been reported before ${ }^{2,4,27}$. We also found isolates harboring $c c r C$, which is similar to that in the USA study ${ }^{3}$. A limitation of our study is that Multiplex
PCR could not able to detect new SCCmec elements due to wide variation in $c c r$ genes. Therefore, sequence-based methods are recommended.

In conclusion, the horizontal transfer of genes between species leads to increases in antibiotic- and disinfectant-resistant genes and the emergence of new types of SCCmec, all of which can influence the clinical features of MRSA in the future. In addition, MRSHo with high antibiotic resistance and unknown $\mathrm{SCC}$ ec type might become a serious problem for the treatment of patients with immune deficiencies, such as children.

\section{Acknowledgments}

This research was supported by the Tehran University of Medical Sciences \& Health Services Grant no. 20839/92-01-30.

\section{Conflict of interest}

The authors declare that have no conflicts of interest.

\section{REFERENCES}

1. Gill VJ, Selepak ST, Williams E C. Species identification and antibiotic susceptibilities of coagulase-negative staphylococci isolated from clinical specimens. J Clin Microbiol. 1983;18(6): 1314-9. 
2. Bouchami O, Ben Hassen A, de Lencastre H, Miragaia M. Molecular epidemiology of methicillin-resistant Staphylococcus hominis (MRSHo): low clonality and reservoirs of SCCmec structural elements. PLoS One. 2011;6(7):e21940.

3. Zhang L, Thomas JC, Miragaia M, Bouchami O, Chaves F, d'Azevedo PA, et al. Multilocus sequence typing and further genetic characterization of the enigmatic pathogen, Staphylococcus hominis. PLoS One. 2013;8(6):e66496.

4. Mendoza-Olazaran S, Morfin-Otero R, Rodriguez-Noriega E, Llaca-Díaz J, Flores-Treviño S, González-González GM, et al. Microbiological and molecular characterization of Staphylococcus hominis isolates from blood. PLoS One. 2013;8(4):e61161.

5. Shore AC, Coleman DC. Staphylococcal cassette chromosome mec: recent advances and new insights. Int J Med Microbiol. 2013;303 (6-7):350-9.

6. Miragaia M, de Lencastre H, Perdreau-Remington F, Chambers HF, Higashi J, Sullam PM, et al. Genetic diversity of arginine catabolic mobile element in Staphylococcus epidermidis. PLoS One. 2009;4(11):e7722.

7. Winn WC, Allen SD, Janda WM, Koneman EW, Procop GW, Schreckenberger PC, et al. Koneman's Color Atlas and Textbook of Diagnostic Microbiology. 6th ed. Philadelphia: Lippincott Williams \& Wilkins; 2006, P: 623-67.

8. Hirotaki S, Sasaki T, Kuwahara-Arai K, Hiramatsu K. Rapid and accurate identification of human-associated staphylococci by use of multiplex PCR. J Clin Microbiol. 2011;49(10):3627-31.

9. Soroush S, Jabalameli F, Taherikalani M, Amirmozafari N, Fooladi AA, Asadollahi K, et al. Investigation of biofilm formation ability, antimicrobial resistance and the staphylococcal cassette chromosome mec patterns of methicillin resistant Staphylococcus epidermidis with different sequence types isolated from children. Microb Pathog. 2016;93:126-30.

10. Clinical and Laboratory Standards Institute. Performance Standards for Antimicrobial Susceptibility Testing; Twenty-Fourth Informational Supplement. CLSI. 2014;34(M100-S24) 68e75.

11. Magiorakos AP, Srinivasan A, Carey RB, Carmeli Y, Falagas ME, Giske CG, et al. Multidrug-resistant, extensively drug-resistant and pandrug-resistant bacteria: an international expert proposal for interim standard definitions for acquired resistance. Clin Microbiol Infect. 2012;18(3):268-81.

12. Caviedes L, Delgado J, Gilman RH. Tetrazolium microplate assay as a rapid and inexpensive colorimetric method for determination of antibiotic susceptibility of Mycobacterium tuberculosis. J Clin Microbiol. 2002;40(5):1873-4.

13. Vali L, Davies SE, Lai LL, Dave J, Amyes SG. Frequency of biocide resistance genes, antibiotic resistance and the effect of chlorhexidine exposure on clinical methicillin-resistant Staphylococcus aureus isolates. J Antimicrob Chemother. 2008; 61(3):524-32.

14. Mohammadi S, Sekawi Z, Monjezi A, Maleki MH, Soroush S, Sadeghifard N, et al. Emergence of SCCmec type III with variable antimicrobial resistance profiles and spa types among methicillin-resistant Staphylococcus aureus isolated from healthcare- and communityacquired infections in the west of Iran. Int J Infect Dis. 2014;25:152-8.
15. Jabalameli F, Mirsalehian A, Khoramian B, Aligholi M, Khoramrooz SS, Asadollahi $\mathrm{P}$, et al. Evaluation of biofilm production and characterization of genes encoding type III secretion system among Pseudomonas aeruginosa isolated from burn patients. Burns. 2012;38(8):1192-7.

16. Ito T, Kuwahara-Arai K, Katayama Y, Uehara Y, Han X, Kondo Y, et al. Staphylococcal Cassette Chromosome mec (SCCmec) analysis of MRSA. Methods Mol Biol. 2014;1085:131-48.

17. Diep BA, Stone GG, Basuino L, Graber CJ, Miller A, des Etages SA, et al. The arginine catabolic mobile element and staphylococcal chromosomal cassette mec linkage: convergence of virulence and resistance in the USA300 clone of methicillin-resistant Staphylococcus aureus. J Infect Dis. 2008; 197(11):1523-30.

18. Fitzgibbon JE, Nahvi MD, Dubin DT, JohnJr JF. A sequence variant of Staphylococcus hominis with a high prevalence of oxacillin and fluoroquinolone resistance. Res Microbiol. 2001;152(9):805-10.

19. Palazzo IC, d'Azevedo PA, Secchi C, Pignatari AC, Darini AL. Staphylococcus hominis subsp. novobiosepticus strains causing nosocomial bloodstream infection in Brazil. J Antimicrob Chemother. 2008;62(6):1222-6.

20. Aligholi M, Mirsalehian A, Halimi S, Imaneini H, Taherikalani M, Jabalameli F, et al. Phenotypic and genotypic evaluation of fluoroquinolone resistance in clinical isolates of Staphylococcus aureus in Tehran. Med Sci Monit. 2011;17(9):71-4.

21. Kratzer C, Rabitsch W, Hirschl AM, Graninger W, Presterl E. In vitro activity of daptomycin and tigecycline against coagulasenegative staphylococcus blood isolates from bone marrow transplant recipients. Eur J Haematol. 2007;79(5):405-9.

22. Gales AC, Jones RN, Andrade SS, Pereira AS, Sader HS. In vitro activity of tigecycline, a new glycylcycline, tested against 1,326 clinical bacterial strains isolated from Latin America. Braz J Infect Dis. 2005;9(5):348-56.

23. Natoli S, Fontana C, Favaro M, Bergamini A, Testore GP, Minelli $\mathrm{S}$, et al. Characterization of coagulase-negative staphylococcal isolates from blood with reduced susceptibility to glycopeptides and therapeutic options. BMC Infect Dis. 2009;9:83.

24. Bjorland J, Steinum T, Kvitle B, Waage S, Sunde M, Heir E. Widespread distribution of disinfectant resistance genes among staphylococci of bovine and caprine origin in Norway. J Clin Microbiol. 2005;43(9):4363-8.

25. de Allori MC, Jure MA, Romero C, de Castillo ME. Antimicrobial resistance and production of biofilms in clinical isolates of coagulase-negative Staphylococcus strains. Biol Pharm Bull. 2006;29(8):1592-6.

26. Garza-González E, Morfin-Otero R, Martínez-Vázquez MA, Gonzalez-Diaz E, González-Santiago O, Rodríguez-Noriega E. Microbiological and molecular characterization of human clinical isolates of Staphylococcus cohnii, Staphylococcus hominis, and Staphylococcus sciuri. Scand J Infect Dis. 2011; 43(11-12): 930-6.

27. Zong Z, Peng C, Lu X. Diversity of SCCmec elements in methicillinresistant coagulase-negative staphylococci clinical isolates. PLoS One. 2011;6(5):e20191. 